\title{
A novel technique: passing through bulky calcified nodules projecting into a popliteal artery using a TruePath crossing device
}

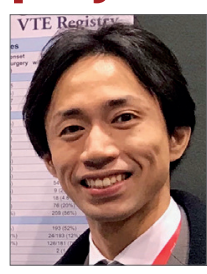

Yuji Nishimoto*, MD; Rei Fukuhara, MD; Masanao Toma, MD; Yukihito Sato, MD, PhD

Department of Cardiology, Hyogo Prefectural Amagasaki General Medical Center, Amagasaki, Japan

This paper also includes supplementary data published online at: www.asiaintervention.org
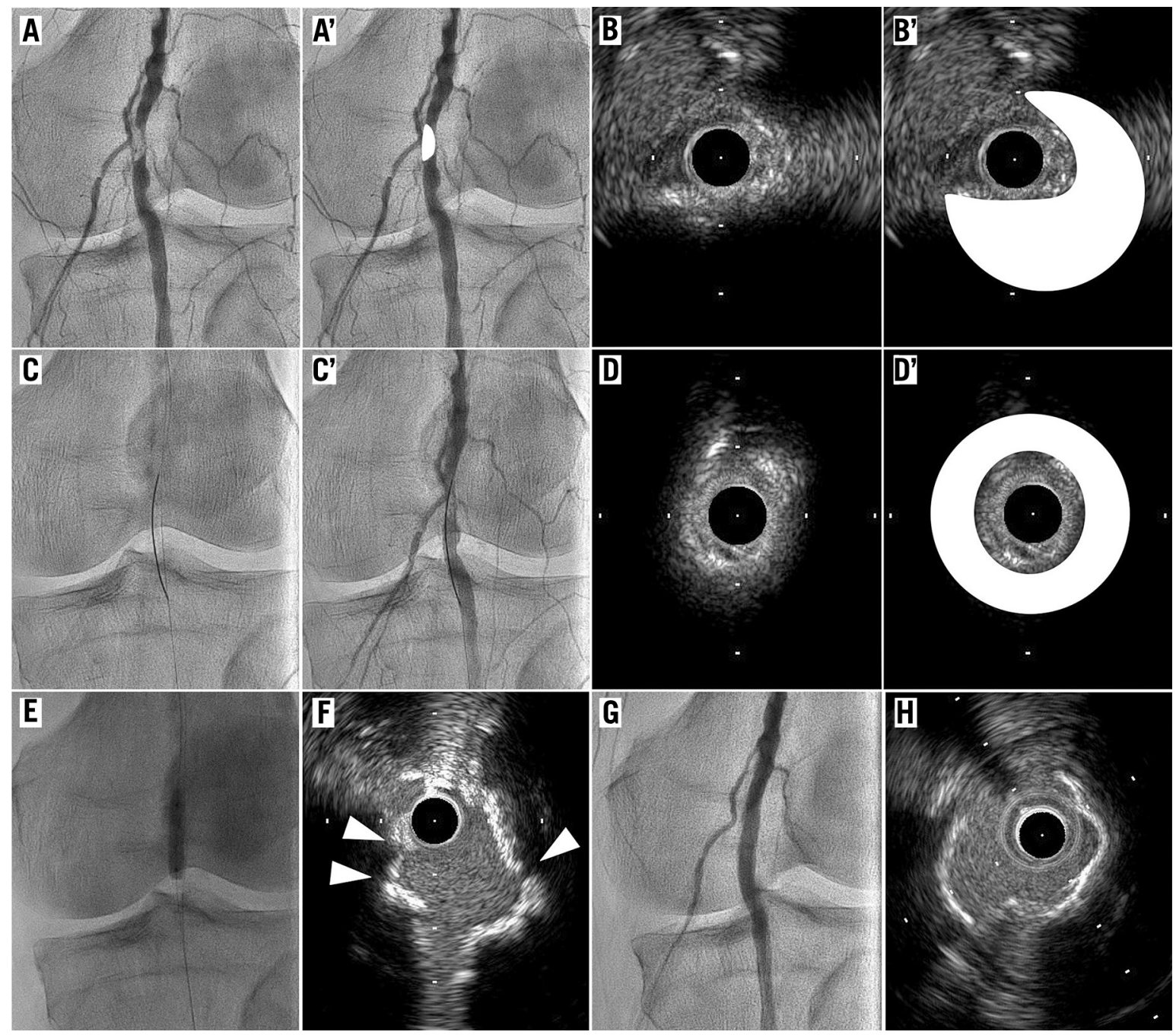

*Corresponding author: Department of Cardiology, Hyogo Prefectural Amagasaki General Medical Center,

2-17-77 Higashinaniwa-cho, Amagasaki 660-8550, Japan.E-mail:yuji.nishimoto@gmail.com 
An 84-year-old man presented with severe eccentric stenosis of the left popliteal artery (Panels A and A', white colour indicates calcification). After crossing the lesion with a tapered tip $1 \mathrm{~g} 0.014$ inch guidewire, and dilatation with a $1.5 \mathrm{~mm}$ balloon, intravascular ultrasound (IVUS) demonstrated eccentric bulky calcified nodules (Panels B and B', white colour indicates calcification; Moving image 1). A conventional larger balloon dilatation in this lumen would have caused an under-expansion, and therefore, we tried a novel CanPath technique: passing through bulky calcified nodules using a TruePath ${ }^{\mathrm{TM}}$ CTO device (Boston Scientific, Marlborough, MA, USA), which consisted of a 0.018 inch wire with a rotating distal diamond-coated tip ${ }^{1}$. The CanPath technique, which is indicated for the longitudinal penetration of calcified nodules using the TruePath CTO device under fluoroscopy guidance, could create an intraluminal pathway. After performing this technique (Panels C and C'), IVUS demonstrated that the wire was located in the middle of the calcification (Panels D and D', white colour indicates calcification; Moving image 2). After dilatation with a $4.0 \mathrm{~mm}$ cutting balloon, the IVUS revealed some cracks in the calcification (Panels E and F, arrowheads indicate cracks). Subsequent to dilatation with a $5.0 \mathrm{~mm}$ balloon, the final angiogram and IVUS demonstrated that the lesion had become well-expanded without a stent implantation (Panels $\mathbf{G}$ and $\mathbf{H}$; Moving image 3). Freedom from any target vessel revascularisation has lasted for 12 months.

The main advantage of the CanPath technique was the increased deliverability of the 0.014 inch system because of the 0.018 inch debulking effect, which should be investigated further. This technique has the limitation of its cost, which requires the TruePath CTO device and IVUS.

\section{Acknowledgement}

We would like to express our gratitude to Mr. John Martin for his grammatical assistance.

\section{Conflict of interest statement}

The authors have no conflicts of interest to declare.

\section{Reference}

1. Bosiers M, Diaz-Cartelle J, Scheinert D, Peeters P, Dawkins KD. Revascularization of lower extremity chronic total occlusions with a novel intraluminal recanalization device: results of the ReOpen study. J Endovasc Ther. 2014;21:61-70.

\section{Supplementary data}

Moving image 1. The initial IVUS in the popliteal artery. Continuous IVUS images pushing forward in the popliteal artery from proximal to distal demonstrate eccentric bulky calcified nodules projecting into the popliteal artery.

Moving image 2. The IVUS after crossing the calcified nodules using the TruePath CTO device. Continuous IVUS images pushing forward in the popliteal artery from proximal to distal show the wire in the middle of the calcification.

Moving image 3. The final IVUS in the popliteal artery. Continuous IVUS images pulling back in the popliteal artery from distal to proximal demonstrate the lesion has become well-expanded without a stent implantation.

The supplementary data are published online at: www.asiaintervention.org 